\title{
Silencing of integrin-linked kinase suppresses in vivo tumorigenesis of human ovarian carcinoma cells
}

\author{
QI LI ${ }^{1}$, CHEN LI ${ }^{2}$, YUN-YAN ZHANG ${ }^{1}$, WEI CHEN ${ }^{1}$, JUN-LI LV $^{1}$, JING SUN $^{1}$ and QING-SHAN YOU ${ }^{1}$ \\ ${ }^{1}$ Department of Gynecology, Harbin Medical University Cancer Hospital, Harbin 150081, Heilongjiang; \\ ${ }^{2}$ Department of Dermatology, Heilongjiang Provincial Hospital, Harbin 150036, Heilongjiang, P.R. China
}

Received October 11, 2012; Accepted January 16, 2013

DOI: $10.3892 / \mathrm{mmr} .2013 .1285$

\begin{abstract}
Integrin-linked kinase (ILK) plays a role in the regulation of multiple cellular functions (e.g., promoting cell migration and proliferation, but inhibiting cell adhesion). This study investigated the inhibitory effects of ILK gene knockdown on the regulation of in vivo tumorigenesis of human ovarian carcinoma cells in nude mouse xenografts. HO-8910 cells were transfected with an ILK antisense oligonucleotide (ILK-ASO) to silence the ILK gene. Expression of ILK mRNA and protein was evaluated by RT-PCR and western blotting, respectively. The cell cycle was assessed by flow cytometric analysis. Cells with or without ILK-ASO transfection were subcutaneously injected into nude mice. The mouse body weight, tumor formation, tumor size and tumor weight were determined up to 30 days after inoculation. Tumor cells transfected with ILK-ASO had significantly decreased ILK mRNA and protein expression $(\mathrm{P}<0.01)$ when compared to the control cells. ILK gene silencing significantly increased the number of cells in the G0/G1 phase (67.61 vs. $43.29 \%$, $\left.\chi^{2}=1197.15, \mathrm{P}<0.01\right)$. After tumor cell inoculation, tumor cells transfected with ILK-ASO showed significantly delayed tumor formation when compared to control $(9.10 \pm 0.74$ vs. $5.30 \pm 0.67$ days, respectively; $\mathrm{P}<0.01)$. In addition, tumor growth was suppressed in the 30 days following inoculation $(\mathrm{P}<0.01$ compared with the controls). The average tumor weight in the ILK-ASO group was statistically lower than that of the control group $(1.29 \pm 0.11$ vs. $1.57 \pm 0.13 \mathrm{~g}$, respectively; $\mathrm{P}<0.01)$. This study demonstrated that ILK-ASO transfection efficiently downregulated ILK expression in human ovarian carcinoma HO-8910 cells and that ILK gene silencing suppressed tumor growth in nude mice xenografts.
\end{abstract}

Correspondence to: Dr Qi Li or Dr Qing-Shan You, Harbin Medical University Cancer Hospital, 150 Haping Road, Harbin 150081, Heilongjiang, P.R. China

E-mail: liqi@ems.hrbmu.edu.cn

E-mail: youqs@sina.com

Key words: integrin-linked kinase, gene silence, ovarian cancer, nude mice xenografts, tumorigenesis

\section{Introduction}

Ovarian cancer is the sixth most common cancer worldwide in women (1). Although the incidence rate varies widely among different geographic regions and ethnic groups, ovarian cancer is more common in industrialized nations, such as Northern Europe and the United States, but it is exceptionally low in Japan (2). The exact cause of ovarian cancer remains unknown. Thus, the mechanisms involved in ovarian epithelial cell malignant transformation remain to be defined. However, hormones, genetic factors, such as mutations of BRCA1 and 2 genes, or family history may play a role in ovarian carcinogenesis. At the molecular level, accumulated data indicate that integrin-regulated cell adhesion, motility and signaling may be involved in ovarian carcinoma cell proliferation (3-6).

Integrin-linked kinase (ILK), a serine-threonine kinase, associates with the cytoplasmic domains of integrin- $\beta 1$ and $-\beta 3(7,8)$. ILK is recognized as a multifunctional intracellular effector of cell-matrix interactions and regulates various important signaling pathways that may be involved in the regulation of tumor cell growth, proliferation, epithelial-mesenchymal transition, migration, invasion, survival and angiogenesis (9). Indeed, the expression of ILK is often upregulated in various human malignancies and is correlated with advanced tumor stage and grade (9). In ovarian cancer, ILK expression was reported to be induced and was found to be associated with tumor progression (10). In addition, inhibition of ILK expression by small molecule inhibitors or RNA interference inhibited the growth of various cancer cells in vitro, including human bladder cancer cells (11), breast cancer cells (12) and hepatocellular carcinoma (HCC) cells (13). In ovarian cancer, knockdown of ILK expression using short hairpin RNA (shRNA) induced growth inhibition and apoptosis of ovarian cancer SKOV3 cells (14). These studies indicate that ILK may be a novel target for cancer therapeutics (15). Nevertheless, prior to clinical translation, the effects of ILK gene silencing on the regulation of tumor formation and growth of ovarian cancer cells need to be established in animal models.

In the present study, we determined the inhibitory effects of ILK gene knockdown on the regulation of tumor formation and growth by inoculating human ovarian cancer HO-8910 cells transfected with an ILK antisense oligonucleotide (ILK-ASO) into nude mice. Our results may provide useful insights into the antitumor activities of ILK gene knockdown. 


\section{Materials and methods}

Reagents. The ILK antisense oligonucleotide (ILK-ASO) sequence (5'-ATGTCGTCCATAGCAGCGTC-3') and the PCR primers were synthesized by the Shanghai Invitrogen Biotechnology Co., Ltd. (Shanghai, China). Lipofectamine $^{\mathrm{TM}} 2000$ transfection reagent was purchased from Invitrogen (Carlsbad, CA, USA). A rabbit polyclonal anti-human ILK antibody was obtained from Cell Signaling Technology, Inc. (Beverly, MA, USA). The cell cycle analysis kit was purchased from Becton-Dickinson (East Rutherford, NJ, USA).

Cell line, culture and oligonucleotide transfection. The human ovarian carcinoma cell line HO-8910 was obtained from the Laboratory of Medical Genetics, Harbin Medical University (Harbin, China). Cells were maintained in Dulbecco's modified Eagle's medium (DMEM) supplemented with 10\% fetal bovine serum (FBS), $100 \mathrm{U} / \mathrm{ml}$ penicillin and $100 \mu \mathrm{g} / \mathrm{ml}$ streptomycin, and incubated at $37^{\circ} \mathrm{C}$ in a $5 \% \mathrm{CO}_{2}$ and $95 \%$ air incubator. Oligonucleotide transfection into cells was carried out as previously described with a few modifications (16). Briefly, cells in the log phase were digested by $0.25 \%$ trypsin (containing $0.02 \%$ EDTA), seeded onto 100 -ml culture flasks and grown for $24 \mathrm{~h}$. Prior to transfection, cells were washed twice with DMEM free of serum and antibiotics. ILK-ASO was mixed with Lipofectamine 2000 in DMEM and incubated for $20 \mathrm{~min}$ at room temperature. This mixture was then added to cells with a final ILK-ASO concentration of $100 \mathrm{nM}$. Cells treated with Lipofectamine 2000 but without ILK-ASO were used as a negative control. After $8 \mathrm{~h}$, the transfection medium was replaced with culture medium for up to 72 days. At the end of the experiments, the cells were subjected to cell viability and gene expression analyses.

Reverse transcription-polymerase chain reaction (RT-PCR). Expression of ILK mRNA in cells was determined by RT-PCR. In brief, $72 \mathrm{~h}$ after oligonucleotide transfection, cells were collected and resuspended at a density of $1 \times 10^{7}$ cells $/ \mathrm{ml}$. Total RNA was collected from $1 \mathrm{ml}$ of the cell suspension and was reverse transcribed using a Promega RT kit (Madison, WI, USA). PCR amplification was then performed using the ILK gene upstream primer (5'-TGGACGACATTTTCACTCAG-3') and downstream primer (5'-CATCAATCATTACACTA CGG-3'), producing an amplified fragment of $984 \mathrm{bp}$. Amplification of the $\beta$-actin gene upstream primer (5'-GCTGGCCGGGACCTGACTGA-3') and downstream primer (5'-AAGCATTTGCGG TGGACGAT-3') produced an amplified fragment with a length of $584 \mathrm{bp}$. PCR amplification conditions were as follows: pre-denaturation at $95^{\circ} \mathrm{C}$ for $5 \mathrm{~min}$ and then 35 cycles of denaturation at $95^{\circ} \mathrm{C}$ for $45 \mathrm{sec}$, annealing at $49^{\circ} \mathrm{C}$ for $45 \mathrm{sec}$, and extension at $72^{\circ} \mathrm{C}$ for $60 \mathrm{sec}$. All protocols had an extra 10 -min extension at $72^{\circ} \mathrm{C}$. Subsequently, PCR products were separated by agarose gel electrophoresis, and then ethidium bromide-stained bands were visualized by ultraviolet transillumination. The fluorescence intensity was quantified using a Gel Imaging System (Kodak, USA). The level of ILK mRNA was semi-quantified by calculating the ILK/ $\beta$-actin ratio. Data were summarized from three independent experiments.
Protein extraction and western blotting. To determine the levels of ILK protein, cells were lysed for $30 \mathrm{~min}$ at $4^{\circ} \mathrm{C}$ and protein was collected and quantified using the Bradford protocol. Denatured protein $(30 \mu \mathrm{g})$ was then separated on acrylamide gels by sodium dodecyl sulfate-polyacrylamide gel electrophoresis (SDS-PAGE) and transferred onto polyvinylidene fluoride (PVDF) membranes. Membranes were blocked, probed with an anti-ILK primary antibody for $1 \mathrm{~h}$ at room temperature, and then blotted with a secondary antibody for another $1 \mathrm{~h}$. Blots were then visualized by 3,3'-diaminobenzidine (DAB) staining. Quantification of western blotting was performed by normalizing the signal intensity of each band to that of the $\beta$-actin control. Data were summarized from three independent experiments.

Cell cycle analysis. Seventy-two hours after transfection, cells were collected, washed with ice-cold phosphate-buffered saline (PBS) and fixed in $70 \%$ ethanol at $4^{\circ} \mathrm{C}$ overnight. The next day, cells were washed with PBS and stained with propidium iodide (PI) in the dark for $30 \mathrm{~min}$ and subjected to FACScan flow cytometry (FC 500MPL, Beckman Coulter, USA) analysis. For data generation, 10,000 cells were analyzed with CXP Acquisition and CXP Analysis software packages (Beckman Coulter).

Nude mouse xenograft assay. Four to six week-old female specific pathogen-free (SPF) nude mice of the BALB/c strain (20-22 g) were purchased from the Vital River Laboratory Animal Technology Co., Ltd. (Beijing, China). Animals were routinely housed and had free access to water and food. All efforts were made to minimize animal suffering and to reduce the number of animals used. All animal procedures and the study were approved by the Ethics Committee of the Harbin Medical University Cancer Hospital. Twenty nude mice were randomly divided into two groups: a control group $(n=10)$ and an ILK-ASO group $(\mathrm{n}=10)$. Animals in the control group were inoculated with negative control cells, while animals in the ILK-ASO group were inoculated with cells transfected with ILK-ASO.

After transfection, tumor cells were collected by centrifugation, and the cell density was adjusted to $1 \times 10^{7}$ cells $/ \mathrm{ml}$. The cell suspension $(0.3 \mathrm{ml})$ was subcutaneously injected into the back of the right shoulder of 10 nude mice (ILK-ASO group). Tumor growth was monitored every day following inoculation. Tumor volume was measured using a caliper on the indicated days after tumor cell injection and was calculated by the following formula: $\mathrm{V}\left(\mathrm{mm}^{3}\right)=\pi \mathrm{x}$ a $\mathrm{x}$ $b^{2} / 6$, where $a$ is the longest diameter of the tumor and $b$ is the shortest diameter of the tumor. Additionally, the body weight of each nude mouse was recorded up to 30 days following inoculation. At the end of the experiments, the animals were sacrificed and tumor tissues were carefully removed and weighed. The tumor growth inhibition rates were determined by the following equations: tumor size inhibition rate (\%) $=\left(1-\mathrm{V}_{\text {ILK-ASO }} / \mathrm{V}_{\text {Control }}\right) \times 100$; and tumor weight inhibition rate $(\%)=\left(1-\mathrm{M}_{\text {ILK-ASO }} / \mathrm{M}_{\text {Control }}\right) \times 100$.

Statistical analysis. Data were analyzed with the Statistical Package for the Social Sciences (SPSS) version 13.0 software (SPSS, Inc., Chicago, IL, USA) and were expressed as the 
A

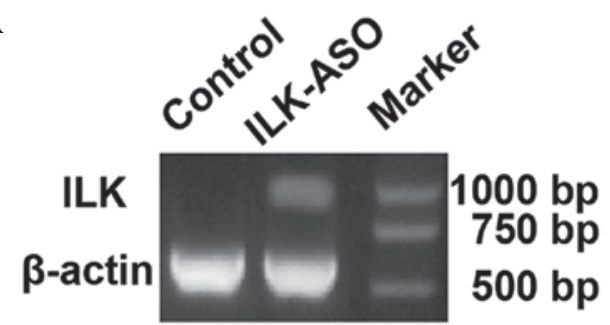

C

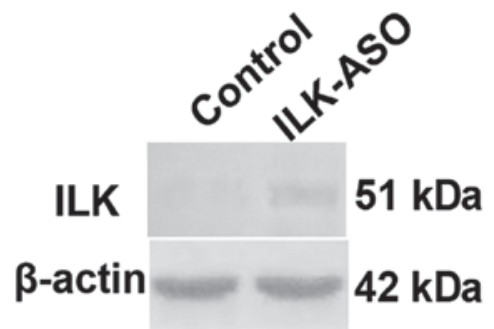

D

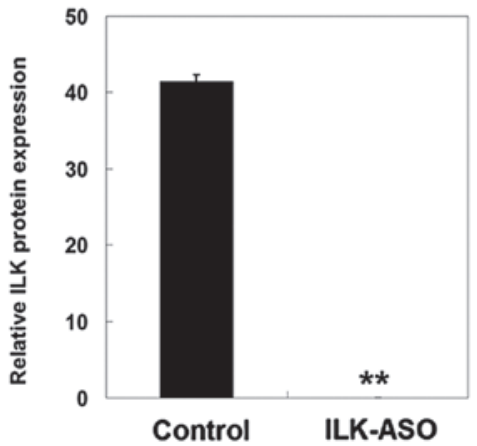

Figure 1. Effect of ILK-ASO transfection on the knockdown of ILK gene expression. Ovarian cancer HO-8910 cells were grown and transiently transfected with ILK-ASO for three days. Total cellular RNA and protein were then isolated and subjected to (A and B) RT-PCR and (C and D) western blot analyses, respectively. Cells without ASO transfection were applied as a control. (B) The levels of ILK mRNA and (D) protein were then normalized to $\beta$-actin. Data were quantified from three independent experiments. ${ }^{* *} \mathrm{P}<0.01$ compared to the controls. ILK, integrin-linked kinase; ASO, antisense oligonucleotide.

A

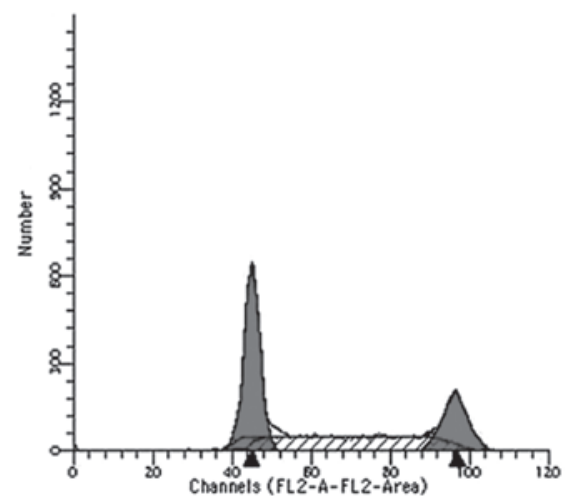

ILK-ASO

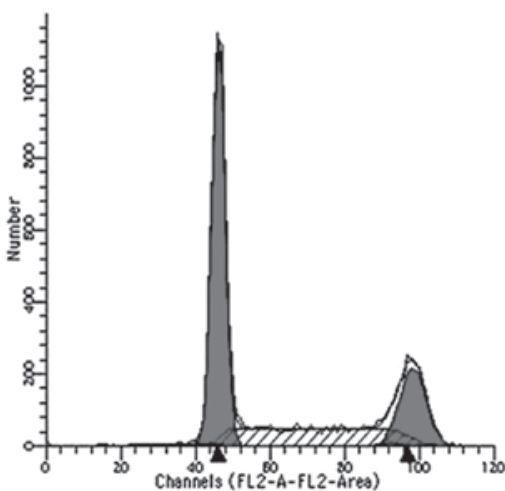

B

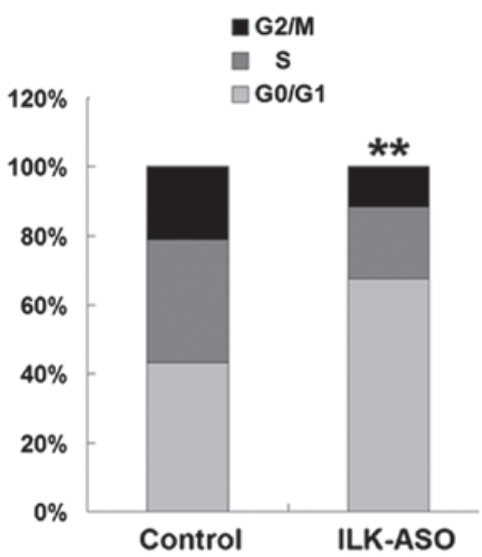

Figure 2. Effect of ILK knockdown on the regulation of HO-8910 cell cycle distribution. (A) Ovarian cancer HO-8910 cells were grown and transiently transfected with ILK-ASO for three days, and then subjected to flow cytometric analyses to determine cell cycle distributions. Cells without ASO transfection were applied as a control. (B) The graph shows the quantified data from the flow cytometry analyses. ${ }^{* *} \mathrm{P}<0.01$ compared to the control. ILK, integrin-linked kinase; ASO, antisense oligonucleotide.

means \pm standard deviation (SD). The Student's t-test was used to compare the quantitative data and the $\chi^{2}$ test was used to analyze enumeration data. $\mathrm{P}<0.05$ was considered to indicate a statistically significant difference.

\section{Results}

Effect of ASO transfection on the knockdown of ILK gene expression. In this study, we first determined the in vitro effect of ILK knockdown on the regulation of tumor growth using ILK-ASO transiently transfected into human ovarian carcinoma HO-8910 cells. The gene silencing efficiency was examined by RT-PCR and western blotting. As shown in Fig. 1, three days after the ASO transfection, the expression of
ILK mRNA and protein was greatly decreased in ILK-ASOtransfected HO-8910 cells as compared with the control cells (ILK mRNA levels, 0 vs. $0.34 \pm 0.01$, respectively; ILK protein levels, 0 vs. $41.44 \pm 0.87$, respectively; $\mathrm{P}<0.01$ ). These results demonstrated that ILK-ASO transfection efficiently downregulated the expression of ILK mRNA and protein in HO-8910 cells.

Effect of ILK knockdown on the regulation of HO-8910 cell cycle distribution. We then observed phenotypic changes of HO-8910 cells after ILK knockdown and performed flow cytometric analyses to determine cell cycle re-distribution. As shown in Fig. 2, the number of cells in the G0/G1 phase was significantly increased as a result of ILK gene silencing 


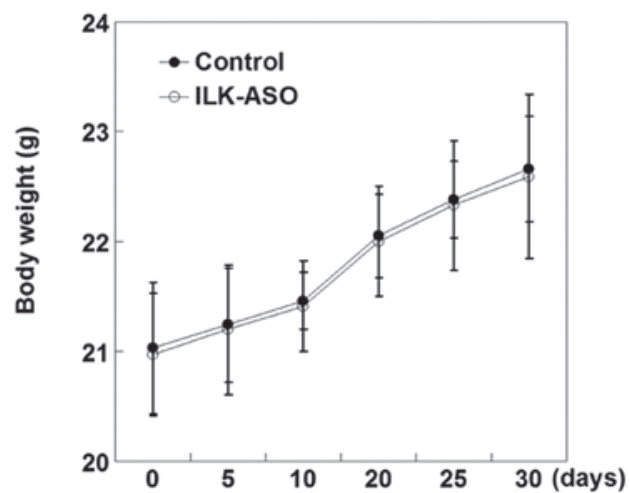

Figure 3. Effect of inoculation of ILK-ASO-transfected HO-8910 cells on the regulation of body weight. Nude mice were inoculated with HO-8910 cells transfected with or without ILK-ASO. Body weight was recorded every five days after tumor cell inoculation for up to 30 days. No significant difference was observed between these two groups of mice.

A

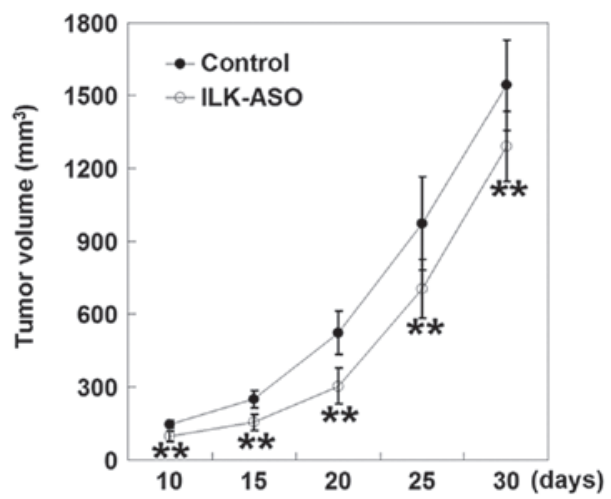

B

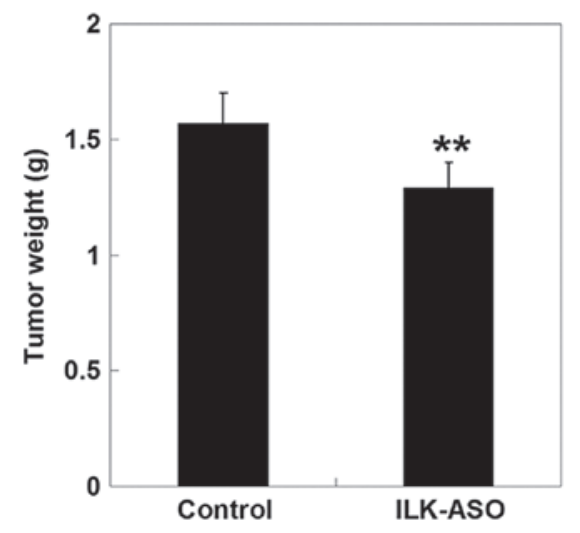

Figure 4. Effect of ILK knockdown on the regulation of tumor formation and growth in nude mouse xenografts. Nude mice were inoculated with HO-8910 cells transfected with or without ILK-ASO. (A) Tumor volume; (B) tumor weight. Tumor weight was measured 30 days after inoculation when the animals were sacrificed.

(67.61 vs. $43.29 \%$ in the control cells; $\chi^{2}=1197.15, \mathrm{P}<0.01$ ). ILK silencing significantly reduced the cell numbers in the $\mathrm{S}$ and $\mathrm{G} 2 / \mathrm{M}$ phase (S phase, 20.69 vs. $35.52 \%$ in the control cells, $\chi^{2}=544.21, \mathrm{P}<0.01 ; \mathrm{G} 2 / \mathrm{M}$ phase, 11.70 vs. $21.19 \%$ in the control cells, $\chi^{2}=327.71, \mathrm{P}<0.01$ ), indicating that ILK silencing inhibits ovarian cancer cell proliferation through G0/G1 phase arrest.
Effect of ILK knockdown on the regulation of tumor formation and growth in nude mouse xenografts. We then performed nude mouse xenograft assays to test the effect of ILK gene knockdown on the regulation of tumor formation and growth in vivo. Our data showed that all of the nude mice survived 30 days following tumor cell inoculation. Moreover, the average mouse body weight gradually increased after tumor cell injection; however, no statistical difference was detected in the body weight between control mice and ILK-ASO-silenced mice at each time point following inoculation ( $\mathrm{P}>0.05$; Fig. 3 ).

Furthermore, a single subcutaneous xenotransplanted mass was formed in each nude mouse after tumor cell inoculation, indicating that ILK gene silencing could not inhibit tumorigenesis in nude mice. However, inoculation of cells transfected with ILK-ASO markedly delayed the tumor formation when compared to control mice $(9.10 \pm 0.74$ days vs. $5.30 \pm 0.67$, respectively, $\mathrm{P}<0.01)$. Thus, the tumor size was markedly decreased in mice injected with tumor cells transfected with ILK-ASO ( $\mathrm{P}<0.01$, Fig. 4). The inhibition rate gradually elevated 10 days after inoculation, peaked at 20 days, and then decreased thereafter (10 days, 33.77\%; 15 days, 37.92\%; 20 days, $41.79 \%$; 25 days, $27.55 \%$; and 30 days, $16.31 \%$ ). Thirty days after tumor cell inoculation, mice were sacrificed and tumor tissues were removed and weighed. The average tumor weight in the ILK-ASO group was statistically lower than that of the control group $(1.29 \pm 0.11$ vs. $1.57 \pm 0.13 \mathrm{~g}$, respectively, $\mathrm{P}<0.01$ ). The tumor weight inhibition rate for mice inoculated with ILK-ASO cells was $17.83 \%$. These observations indicated that inoculation of cells transfected with ILK-ASO suppressed tumor growth in vivo.

\section{Discussion}

ILK protein is a unique intracellular adaptor and kinase that links the cell-adhesion receptors, integrins and growth factors to the cytoskeleton. In addition, ILK regulates a variety of intracellular signaling pathways (15). Thus, ILK plays an essential role in the regulation of various cellular processes that are critical for tumor progression, including tumor cell proliferation, migration, invasion and survival, epithelialmesenchymal transition and angiogenesis (9). Moreover, the regulatory activity of ILK protein is controlled by a network of intracellular and intercellular processes that result in aberrant ILK expression and signaling in a number of human malignancies (17). This study explored the role of ILK in mediating tumor cell proliferation in human ovarian carcinoma by using ILK-ASO. ASO is an established and mature technique in molecular biology, which is designed to complementarily bind to specific mRNA, resulting in the degradation of the message encoding the targeted protein (18). In particular, this method may be used as a novel therapeutic tool in preclinical or future clinical research. In this present study, an ASO specifically targeting against ILK was synthesized and transfected into human ovarian carcinoma HO-8910 cells. Our data showed that three days after transfection, expression of ILK mRNA and protein was markedly downregulated in HO-8910 cells, indicating the successful silencing of ILK gene expression.

A previous study demonstrated that overexpression of ILK protein promoted anchorage-independent cell cycle progression (19). In this study, we knocked down ILK expression and 
flow cytometric analyses revealed that ILK silencing inhibited tumor cell proliferation via G0/G1 phase arrest in HO-8910 cells. Our current data are in accordance with a previous report, which demonstrated that transfection of a kinase-deficient, dominant-negative form of ILK induced G1 phase cycle arrest and enhanced tumor cell apoptosis in PTEN-mutant prostate cancer cells (20). Taken together, these observations indicate that ILK-induced tumor cell proliferation was mediated by cell cycle progression.

Since silencing of the ILK gene by shRNA induced growth inhibition and apoptosis in ovarian cancer SKOV3 cells (14), we aimed to determine the inhibitory effects of ILK gene silencing on tumorigenesis in vivo. HO-8910 cells transfected with ILK-ASO or without transfection (control) were subcutaneously injected into nude mice. Inoculation of tumor cells transfected with ILK-ASO significantly delayed tumor formation and suppressed tumor growth. These findings are consistent with previous reports in other types of human cancer. For example, Chan et al (13) suppressed tumorigenesis in vivo after subcutaneously injecting ILK knockdown HCC cells into the right flank of nude mice. Moreover, nude mice injected with bladder BIU-87 cells, which were transfected with ILK small interference RNA (siRNA), showed a significant inhibition in tumor growth, as well as decreased tumor weight and microvessel density and an increased apoptosis rate when compared with the control groups (11). However, to date, the underlying molecular mechanisms of ILK silencing-induced tumor growth suppression are poorly understood. Activation of Akt $(11,13)$, glycogen synthase kinase $3-\beta$ (GSK-3 $\beta)$ (11), or other unknown pathways may contribute to this process.

In our current study, it should be noted that tumor size inhibition gradually elevated 10 days after inoculation, peaked at 20 days and decreased thereafter, implying that the degradation of ASO may have resulted in the failure of growth suppression 20 days after inoculation. Thus, ASO degradation at later time points may be a limitation of this study. In order to yield a better therapeutic outcome, more frequent administration of ASO may be required in future studies.

Although encouraging results from preclinical and clinical studies have been obtained and significant progress has been made in developing ASO as an antitumor drug, it is not yet recognized as an effective therapeutic (21). Future studies should continue to compare the anti-tumorigenic capabilities between ASO and RNA interference techniques, including siRNA and shRNA.

\section{Acknowledgements}

This study was supported in part by a grant from the Natural Science Foundation of Heilongjiang Province (QC 2010067).

\section{References}

1. Notani P: Global variation in cancer incidence and mortality. Curr Sci 81: 465-475, 2001

2. Holschneider $\mathrm{CH}$ and Berek JS: Ovarian cancer: epidemiology, biology, and prognostic factors. Semin Surg Oncol 19: 3-10, 2000 .
3. Cannistra SA, Ottensmeier C, Niloff J, Orta B and DiCarlo J: Expression and function of beta 1 and alpha v beta 3 integrins in ovarian cancer. Gynecol Oncol 58: 216-225, 1995.

4. Cruet-Hennequart S, Maubant S, Luis J, Gauduchon P, Staedel C and Dedhar S: alpha(v) integrins regulate cell proliferation through integrin-linked kinase (ILK) in ovarian cancer cells. Oncogene 22: 1688-1702, 2003.

5. Ruseva Z, Geiger PX, Hutzler P, Kotzsch M, Luber B, Schmitt M, Gross E and Reuning U: Tumor suppressor KAI1 affects integrin alphavbeta3-mediated ovarian cancer cell adhesion, motility, and proliferation. Exp Cell Res 315: 1759-1771, 2009.

6. Kaur S, Kenny HA, Jagadeeswaran S, Zillhardt MR, Montag AG, Kistner E, Yamada SD, Mitra AK and Lengyel E: \{beta\}3integrin expression on tumor cells inhibits tumor progression, reduces metastasis, and is associated with a favorable prognosis in patients with ovarian cancer. Am J Pathol 175: 2184-2196, 2009.

7. Mulrooney J, Foley K, Vineberg S, Barreuther M and Grabel L: Phosphorylation of the betal integrin cytoplasmic domain: toward an understanding of function and mechanism. Exp Cell Res 258: 332-341, 2000.

8. Dedhar S, Williams B and Hannigan G: Integrin-linked kinase (ILK): a regulator of integrin and growth-factor signalling. Trends Cell Biol 9: 319-323, 1999.

9. McDonald PC, Fielding AB and Dedhar S: Integrin-linked kinase - essential roles in physiology and cancer biology. J Cell Sci 121: 3121-3132, 2008.

10. Ahmed N, Riley C, Oliva K, Stutt E, Rice GE and Quinn MA: Integrin-linked kinase expression increases with ovarian tumour grade and is sustained by peritoneal tumour fluid. J Pathol 201: 229-237, 2003.

11. Gao J, Zhu J, Li HY, Pan XY, Jiang R and Chen JX: Small interfering RNA targeting integrin-linked kinase inhibited the growth and induced apoptosis in human bladder cancer cells. Int J Biochem Cell Biol 43: 1294-1304, 2011.

12. Pontier SM, Huck L, White DE, Rayment J, Sanguin-Gendreau V, Hennessy B, Zuo D, St-Arnaud R, Mills GB, Dedhar S, et al: Integrin-linked kinase has a critical role in ErbB2 mammary tumor progression: implications for human breast cancer. Oncogene 29: 3374-3385, 2010.

13. Chan J, Ko FC, Yeung YS, Ng IO and Yam JW: Integrin-linked kinase overexpression and its oncogenic role in promoting tumorigenicity of hepatocellular carcinoma. PLoS One 6: e16984, 2011.

14. Liu Q, Xiao L, Yuan D, Shi X and Li P: Silencing of the integrinlinked kinase gene induces the apoptosis in ovarian carcinoma. J Recept Signal Transduct Res 32: 120-127, 2012.

15. Hannigan G, Troussard AA and Dedhar S: Integrin-linked kinase: a cancer therapeutic target unique among its ILK. Nat Rev Cancer 5: 51-63, 2005.

16. Yang Y, Fukui K, Koike T and Zheng X: Induction of autophagy in neurite degeneration of mouse superior cervical ganglion neurons. Eur J Neurosci 26: 2979-2988, 2007.

17. McDonald PC and Dedhar S: The role of integrin-linked kinase in cancer development and progression. In: Cell-Extracellular Matrix Interactions in Cancer.Zent R and Pozzi A (eds). Springer, New York, pp245-273, 2010.

18. Monteith DK, Geary RS, Leeds JM, Johnston J, Monia BP and Levin AA: Preclinical evaluation of the effects of a novel antisense compound targeting C-raf kinase in mice and monkeys. Toxicol Sci 46: 365-375, 1998.

19. Radeva G, Petrocelli T, Behrend E, Leung-Hagesteijn C, Filmus J, Slingerland J and Dedhar S: Overexpression of the integrin-linked kinase promotes anchorage-independent cell cycle progression. J Biol Chem 272: 13937-13944, 1997.

20. Persad S, Attwell S, Gray V, Delcommenne M, Troussard A, Sanghera J and Dedhar S: Inhibition of integrin-linked kinase (ILK) suppresses activation of protein kinase B/Akt and induces cell cycle arrest and apoptosis of PTEN-mutant prostate cancer cells. Proc Natl Acad Sci USA 97: 3207-3212, 2000.

21. Rayburn ER and Zhang R: Antisense, RNAi, and gene silencing strategies for therapy: mission possible or impossible? Drug Discov Today 13: 513-521, 2008. 